\title{
The conceptual similarity effect in free recall
}

\author{
JOHN C. KOEPPEL, UNIVERSITY OF NORTH DAKOTA
} ROBERT S. BEECROFT, UNIVERSITY OF IOWA

A sizable facilitative effect of conceptual similarity on free recall was obtained with a mixed-list technique. Subsequent to the first recall, related and unrelated words were learned at the same rate.

Conceptual similarity is one kind of intralist similarity analyzed in verbal learning. Although a high degree of intralist similarity generally interferes with serial and paired-associate learning, it usually facilitates free recall. This may be illustrated by Underwood's (1964, pp. 62-65) analysis of conceptual similarity and free recall which was the stimulus for our work. Underwood used four lists of 16 words each. After each list was read orally to a group, the Ss wrote down the words they could recall. Each list was presented and recalled once. In two of the lists the words were unrelated to each other. For example, one list was APPLE, FOOTBALL, EMERALD, TROUT, etc. This was a low similarity list. The other two lists were made up of four sets of four conceptually related words. For example, one set was TANGO, WALTZ, FOXTROT, and RUMBA. Underwood found that for lists of unrelated words recall averaged 11 out of 16 items; for lists of related words 14 out of 16 was the average recall.

We had mixed feelings about the results. It seemed to us that the conceptual similarity effect probably was underestimated since the 11 out of 16 average recall for unrelated words left little room for improvement. At the same time, we felt that having four sets of related words in a 16-item list which contained no unrelated words perhaps made the conceptual relationship too obvious to the Ss. We wondered what would happen if the two types of lists were mixed together with the related words well-dispersed in a lengthy mixed list.

\section{Method}

A single list of 64 words was presented individually. These were the words used by Underwood, with a few changes which were made because pooling the four lists created some related pairs. The list was presented by means of a memory drum, each turn of which displayed a line of four words. There were 16 lines in all; each line was presented for $4 \mathrm{sec}$. In the list there were 32 unrelated words and 32 related words in eight sets of four each. The related words in any one set were scattered so that no two related words appeared in the same column or the same one-fourth of the list. It took $64 \mathrm{sec}$ to present the list and as soon as the last line disappeared from view $S$ began oral recall. Six such presentations and recalls were made. The words appeared in the same 4 by 16 arrangement on all trials. There is a serial position effect which is largely limited to the first and last lines of the matrix. If the serial order of the items is changed from one trial to the next, words tend to be recalled after they move into the favored eight positions. This would selectively favor unrelated words.

The Ss were 20 female students recruited from general psychology classes at Oklahoma State University. The study was a replication of an experiment conducted by members of an experimental psychology class which yielded closely comparable results.

\section{Results}

The conceptual similarity effect appeared in the first recall. The average recall was 14 words, of which 11 were related and three were unrelated. This is a much larger effect than Underwood achieved. If the unrelated words in the first and last lines are disregarded, then no unrelated word was recalled by more than $20 \%$ of the Ss. Some familiar words such as GASOLINE, PICTURE, BUS, and DOCTOR were not recalled by any $S$. The median number of Ss recalling the typical unrelated word was one. Recalls of the related words were more variable. They ranged from zero to $60 \%$. The median number of Ss recalling the typical related word, again with the first and last lines of the matrix disregarded, was six.

Recall over the six trials is shown in Fig. 1. Recall of both related and unrelated words increased over trials to a total of almost 50 words on the sixth recall. The curves are almost exactly parallel for the first four trials, although probably because it approaches the ceiling the related curve breaks first. Discussion

The mixed-list technique revealed a large effect of conceptual similarity on first recall. The ratio of recall of related to unrelated words was approximately

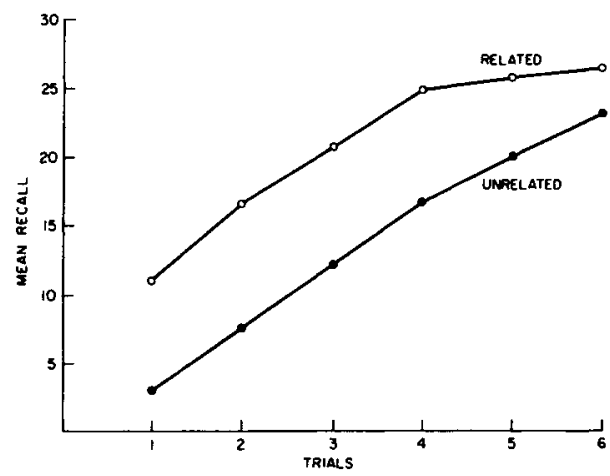

Fig. 1. Mean recall of related and unrelated words as a function of trials. 
four to one. If the concept is the unit of memory, as Underwood and others have suggested, this is what one would expect. Subsequent to the first recall, learning of related and unrelated words seemingly occurred at the same rate. This is difficult to reconcile with the concept-learning hypothesis, for, depending on assumptions concerning memorization, the curves ought to diverge or converge within a few trials. Dual linear functions are most compatible with a free recall model which permits replacement of alreadyrecalled items in the to-be-learned pool for the next trial. They may be more likely to be learned again, but must be replaced to generate such near-linear functions.

\section{References}

UNDERWOOD, B. J. The representativeness of rote verbal learning. In A. W. Melton (Ed.), Categories of human learning. New York: Academic Press, 1964. 